\title{
matters \\ Destabilisation of Argonaute 2 generates truncated protein: halfAgo2
}

Sarah Bajan, Michael Johnston, Gyorgy Hutvagner

School of Biomedical Engineering, University of Technology Sydney; Research and Development, Hutman Diagnostics AG $\checkmark$ Correspondence Sarah.Bajan@uts.edu.au

8 Disciplines Molecular Biology Biochemistry

Q Keywords Argonaute Proteins MicroRNA

RISC

Argonaute 2

Argonaute 2 Stability

i- Type of Observation Standalone

\& Type of Link

Standard Data

(C) Submitted Oct 24, 2018 (-) Published Nov 13, 2018

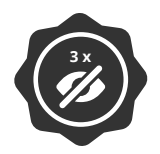

Triple Blind Peer Review The handling editor, the reviewers, and the authors are all blinded during the review process.

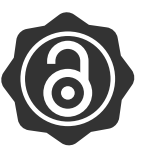

Full Open Access

Supported by the Velux Foundation, the University of Zurich, and the EPFL School of Life Sciences.

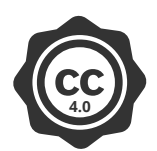

Creative Commons 4.0 This observation is distributed under the terms of the Creative Commons Attribution 4.0 International License.

\begin{abstract}
The Argonaute 2 (Ago2) protein is an essential effector protein in miRNA-mediated mechanisms that regulate gene expression. Ago2 directly binds to the miRNA, forming the RISC. RISC function is critical to controlling key biological processes and when dysregulated can result in disease pathogenesis. Understanding Ago2 protein stability and turnover will further our understanding in how RISC function is regulated. In human cells, we discovered a previously unidentified $\sim 55 \mathrm{kDa}$ protein that is a truncated form of Agoz, that is formed from proteolytic cleavage of the full length Agoz protein. Further experiments are needed to determine (i) the detailed mechanism that forms halfAgo2 (ii) the cellular or environmental triggers or stresses that initiate halfAgo2 production and (iii) if halfAgo2 has a potentially new role in gene regulation.
\end{abstract}

\section{Introduction}

miRNAs are endogenous small molecules that are essential regulators of human development. miRNAs function by targeting the post-transcriptional stages of gene expression, via several distinct mechanisms [1]. miRNA regulatory function depends on the miRNA directly binding to an Argonaute (Ago) protein, forming the RNA Induced Silencing Complex (RISC). In this complex the miRNA acts a guide, by binding to a complementary site within mRNA and bringing the RISC, and its associated regulatory proteins, to the target [2] [3].

RISC regulates key biological processes, therefore any disruption to RISC function can have severe consequences, and misregulation of RISC is implicated in the development of disease [4]. It is therefore important that the stability of RISC and accordingly its components, miRNA and Ago, are controlled. There are several known mechanisms that mediate miRNA stability and turnover, including homeostatic and feedback mechanisms that coordinate miRNA levels with Ago levels [5] [6] [7] [8]. However, we have only limited understanding into mechanisms that regulate the turnover of Ago proteins and the RISC complex.

There is emerging evidence that Ago function and stability is mediated by a variety of post-translational modifications of the protein, which occurs as a consequence of complex signalling pathways [9] [10] [11] [12] [13]. These modifications can alter protein function, stability, and localisation. Depending on the modification, these changes can be permanent or reversible. Therefore Ago levels are potentially highly dynamic and are responsive to internal and external stimuli.

While the 4 human Ago proteins (1-4) display some functional redundancy, Agoz is the most abundant in commonly used human cell lines [14] and the most studied in miRNA regulation.

\section{Objective}

While analysing the protein expression of full-length (FL), endogenous, Ago2 ( $85 \mathrm{kDa})$ in HeLa cell lysate, we observed that an Ago2-specific monoclonal antibody targeted to the N-terminus of the protein [15], also bound to a previously unidentified protein of approximately $55 \mathrm{kDa}$. The objective of this study was to investigate this $55 \mathrm{kDa}$ protein, and to test our hypothesis that this protein is a truncated form of FL human Agoz. 
A

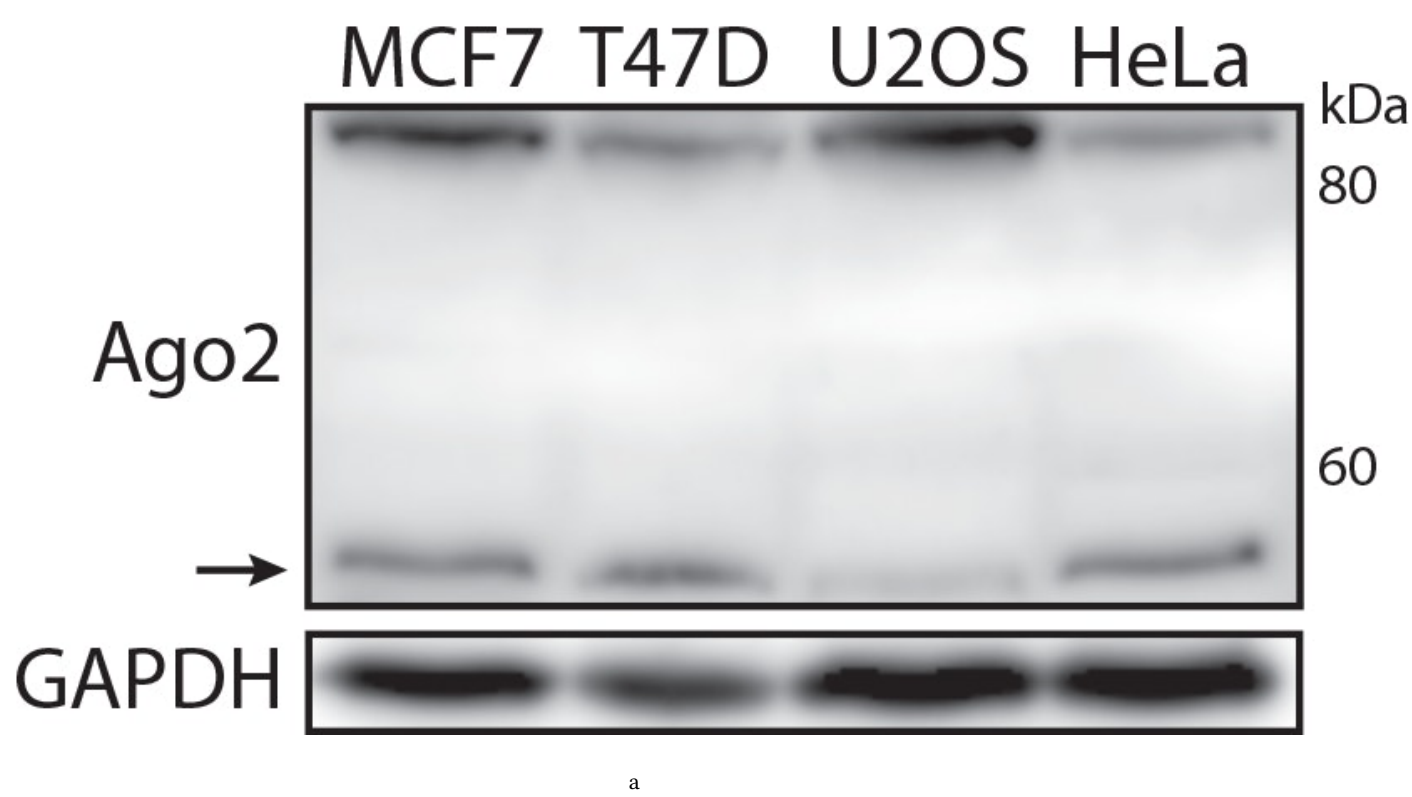

B
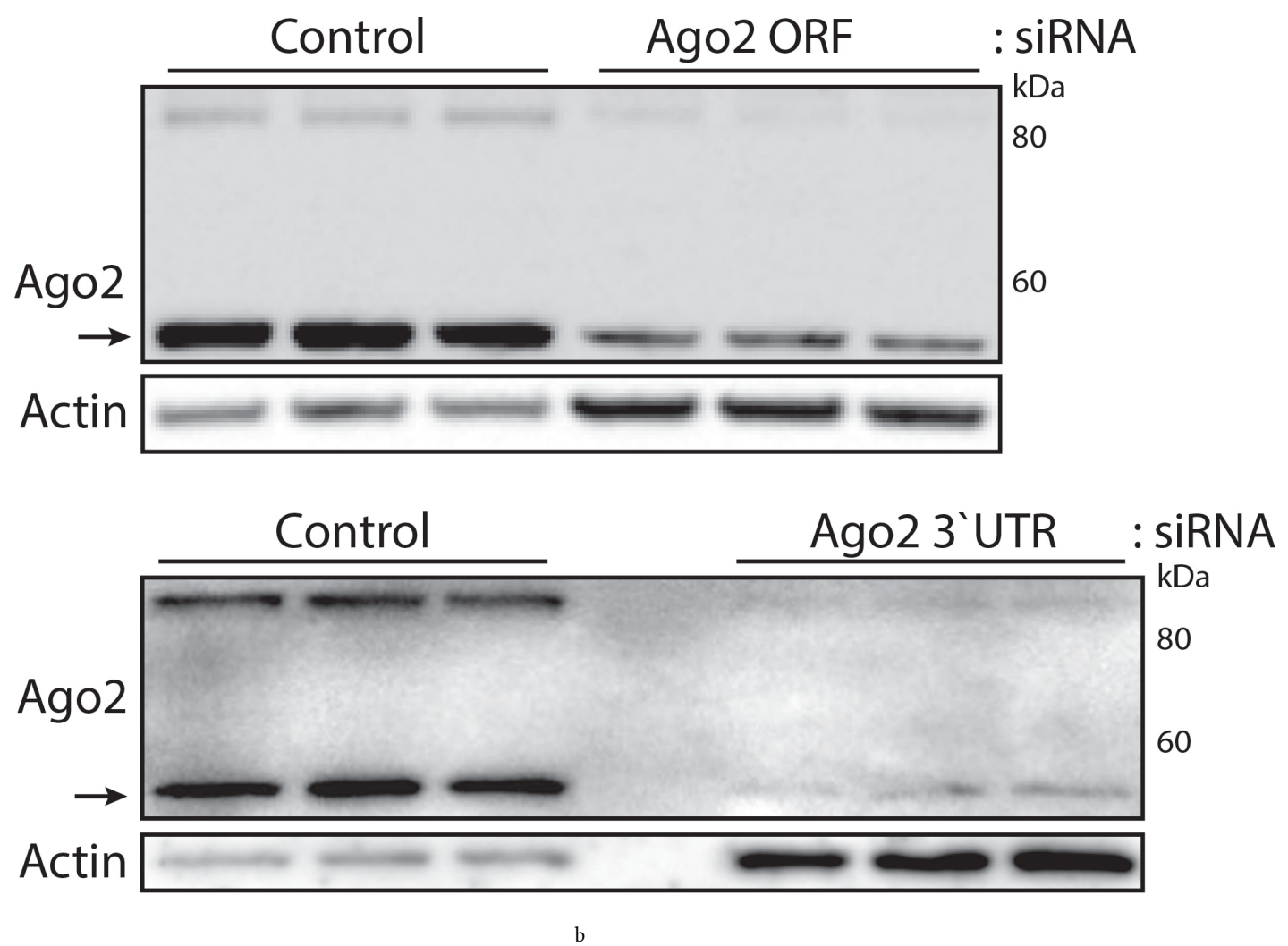
C

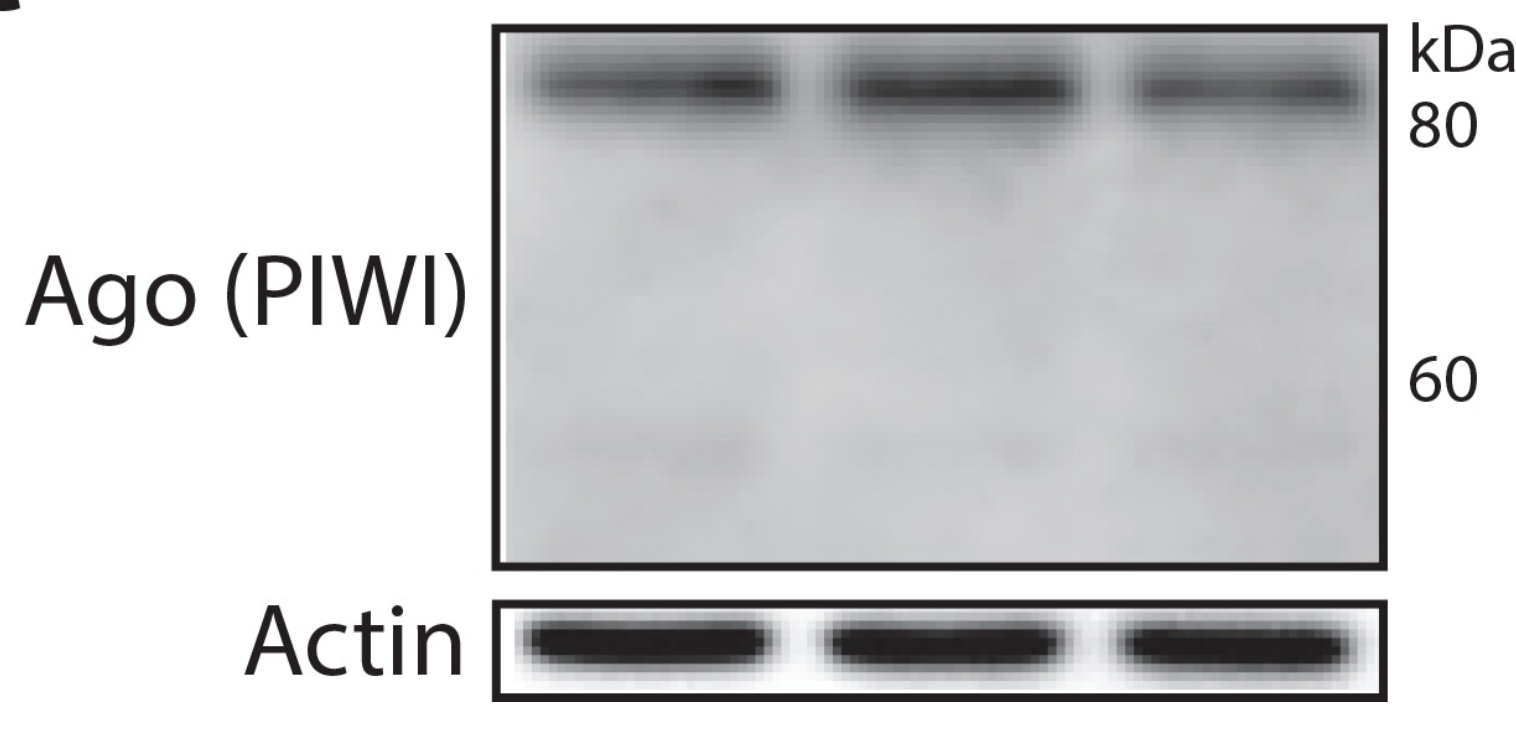

D

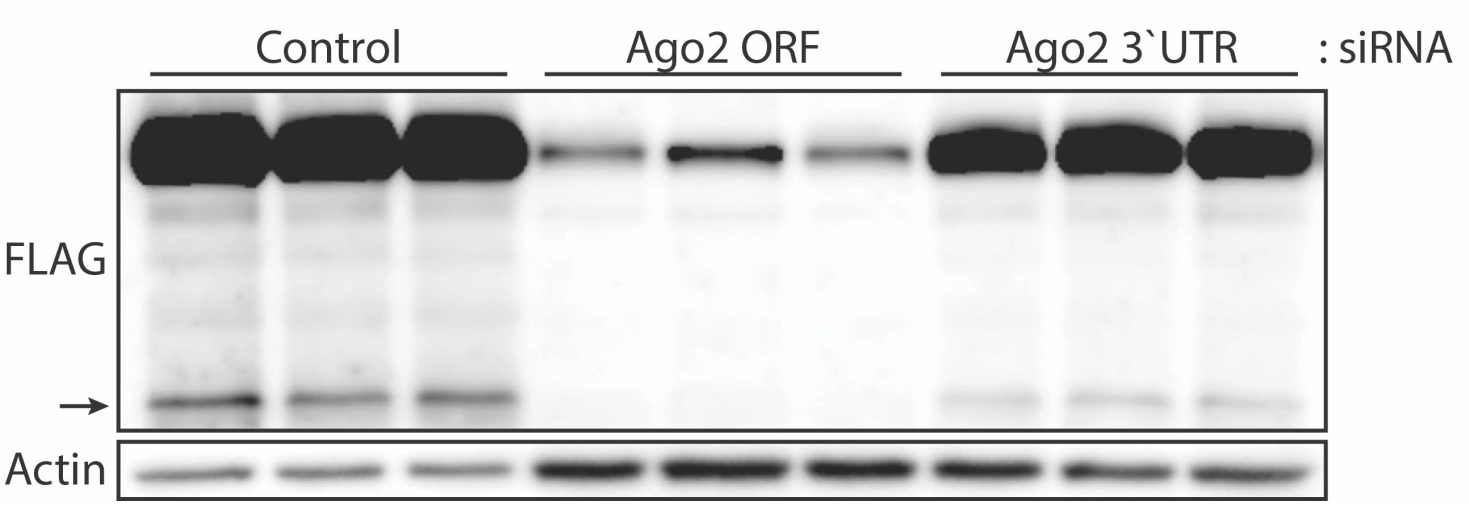

$\mathrm{d}$ 


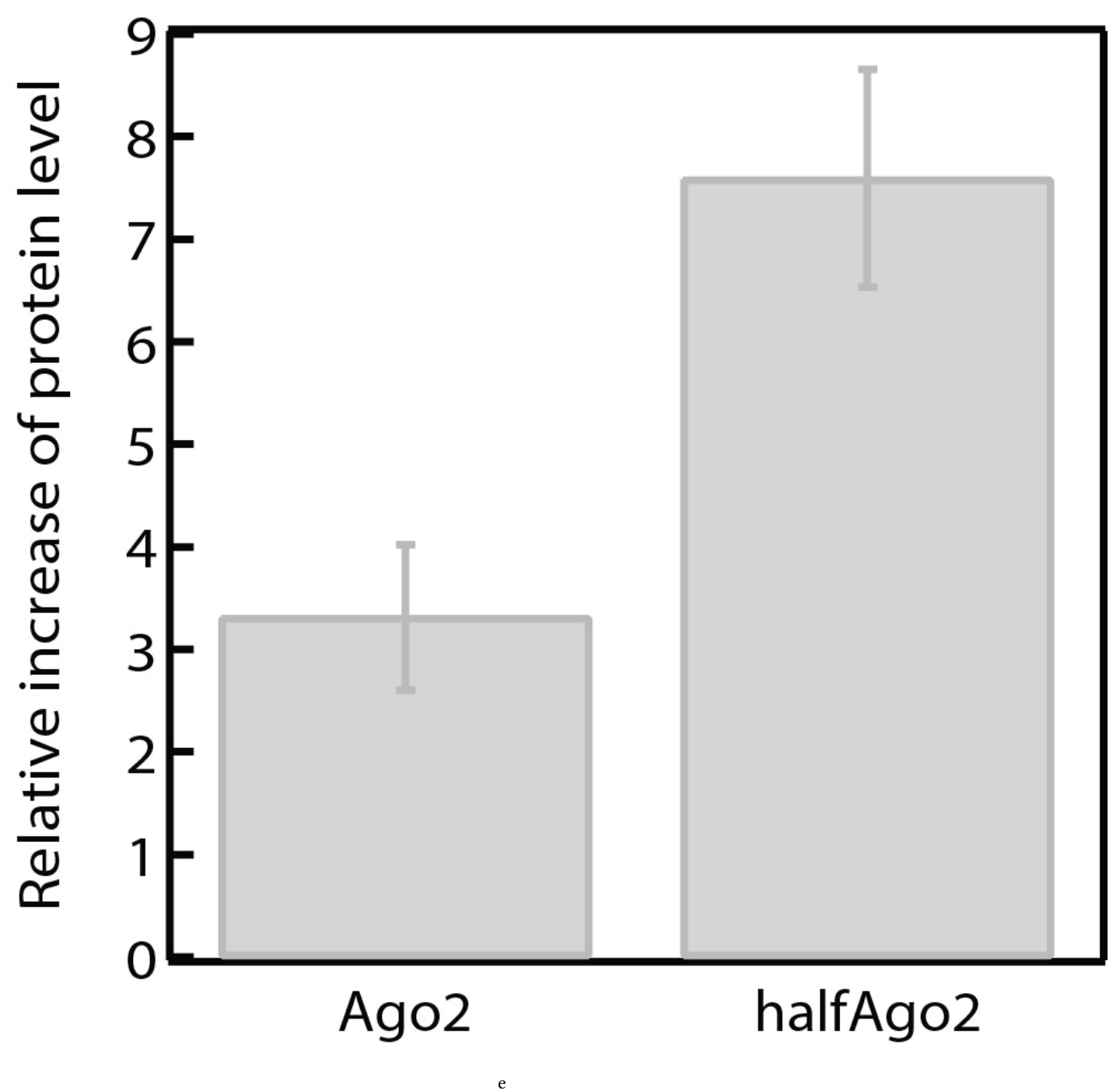

Figure Legend

Figure 1. Endogenous and recombinant Ago2 can form a stable, truncated protein: halfAgo2.

(A) Full length (FL) Ago2 ( $\sim 5 \mathrm{kDa})$ and halfAgo2 ( $\sim 55 \mathrm{kda}$; indicated by the arrow) are expressed in a variety of immortalized human cell lines.

(B) HeLa cells treated with siRNA that specifically targets the Agor open reading frame/coding sequence (Ago2 ORF) decreases expression of both FL Ago2 and halfAgo2.

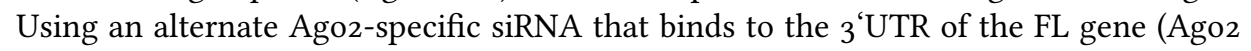

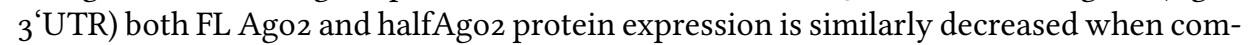
pared to control.

(C) halfAgo2 does not contain the PIWI domain. The expression of Ago proteins in HeLa lysate was tested with an antibody that specifically binds to the C-terminal PIWI domain. Only FL Ago proteins were visible.

(D) Transiently expressed N-terminally tagged FLAG::Ago2 also produced a stable, truncated N-terminal Ago2 product. The expression of FLAG::halfAgo2 is inhibited when treated with an siRNA that can target this plasmid by targeting the Agoz ORF, but is unaffected when treated with an siRNA that cannot target the plasmid (siRNA targeting Ago2 3 'UTR).

(E) FL Ago2 and halfAgo2 protein levels accumulate when the proteasome is inhibited by MG132 treatment in HeLa cells. Expression normalised to DMSO control $(n=3)$. 


\section{Results \& Discussion}

We initially observed the unidentified $55 \mathrm{kDa}$ protein, predicted to be a truncated Ago2 protein which includes the $\mathrm{N}$-terminus of the FL Agoz protein, in HeLa cells. To ensure that this phenomenon was not cell line specific, we tested several human immortalised cell lines, derived from a variety of tissues (breast, bone and cervix) (Fig. 1A, $\mathrm{S}_{1}$ ). We detected the $55 \mathrm{kDa}$ band in the majority of all samples tested, with variable expression levels between cell lines. As other proteins tested in these lysates show no signs of degradation, we can also conclude that the $55 \mathrm{kDa}$ protein is not due to general degradation of the cell lysate (Fig. S1). These data suggests the production of the $55 \mathrm{kDa}$ protein is a regulated process, with a potential physiological function.

To eliminate the possibility that the $55 \mathrm{kDa}$ band is a non-specific band cross-reacting with the N-terminal targeting Agoz antibody, we wished to see if the expression of the $55 \mathrm{kDa}$ band was dependent on FL Agoz protein expression. We used an siRNA that specifically targets the open reading frame (ORF) of the Ago2 transcript, which will reduce FL Ago2 protein expression level. When FL Ago2 protein expression was knocked down, we observed that the $55 \mathrm{kDa}$ protein expression levels also decreased when compared to the control samples (Fig. 1B). To confirm this result we repeated the Ago2 knockdown with an independent siRNA that targeted a different segment of the FL Ago2 transcript, the 3 'UTR. This experiment produced similar results, with reduced protein expression of both the FL Ago2 and the $55 \mathrm{kDa}$ protein, suggesting this effect is not due to off-target effects of the siRNAs used. These data indicate that FL Agoz RNA production is required for the $55 \mathrm{kDa}$ protein to be present. Furthermore, there are no identified alternatively spliced Ago2 isoforms that include the 3 'UTR sequence of the FL Ago2 transcript. This suggests the truncated protein is not a product of alternative splicing.

Due to the size of this protein $(\sim 55 \mathrm{kDa})$ and that it contains the N-terminal domain of Ago2, we predicted that this truncated Ago2 does not contain the PIWI domain (required for target cleavage and binding the $5^{6}$ end of mature miRNAs) present in the FL Ago2, as this domain is located near the $\mathrm{C}$-terminus of the protein. To investigate this we used an antibody that targets the PIWI domain of human Ago proteins (Fig. 1C). This antibody only recognises FL Ago proteins, confirming that the $55 \mathrm{kDa}$ truncated protein does not contain the PIWI domain, and therefore potentially functions independently of binding miRNAs. This data suggests that after FL Agoz cleavage, the N-terminal containing polypeptide (halfAgo2) is more stable than the C-terminal containing product, suggesting halfAgo2 has an independent function. The N-terminal domain of Ago2 has an essential role in separating the strands of miRNA duplexes [16], while the adjacent

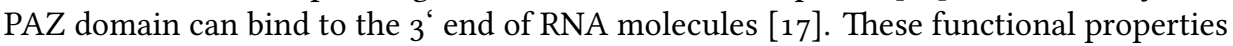
indicate that halfAgoz could play a role in independent gene regulatory mechanisms. To further investigate the origin of the $55 \mathrm{kDa}$ protein, we transiently expressed a FLAGtagged FL Ago2 gene expression plasmid into HeLa cells. The FLAG tag is situated the $\mathrm{N}$-terminus of the protein, and therefore the tag will be present in a truncated product derived from the exogenous FL Ago2 that includes the N-terminal domain. This plasmid contains the endogenous FL Ago2 ORF, but contains plasmid specific sequences upstream and downstream of the Agoz coding sequence. When examining the lysate for FLAG-tagged proteins we also observed a FLAG-tagged truncated $55 \mathrm{kDa}$ protein (Fig. 1D). When samples expressing the FLAG-Agoz plasmid were also transfected with an siRNA that targets the Ago2 ORF, and could therefore target the FLAG-Ago2 transcript, expression of both the FLAG-tagged FL Ago2 and the smaller 55kDa protein was reduced. However, neither of their expression was altered when treated with the Ago2

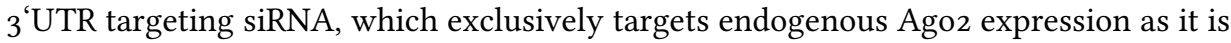
incapable of binding to the FLAG-Ago2 transcript (Fig. 1D).

As the expression of the exogenous, FLAG-tagged Ago2 is derived from a cDNA sequence of the FL Ago2 transcript that has been inserted into an expression vector, the sequence does not contain introns. Therefore, FLAG-Ago2 expression is independent of splicing mechanisms. As FLAG-halfAgoz expression is observed, this further suggests that this protein is not the result of alternative splicing, but may instead be formed from proteolytic cleavage of the FL Ago2 protein. We can conclude from this data that the 55 $\mathrm{kDa}$ Ago2 band is most likely derived from a post-translational mechanism that targets 
the FL Ago2 protein. We will refer to this Ago2 protein as halfAgo2.

We investigated if, like FL Ago2, degradation of the $55 \mathrm{kDa}$ Agoz protein is mediated by the $26 \mathrm{~S}$ proteasome [18]. We treated HeLa cells with the proteasome inhibitor MG132 and found that both FL Ago2 and the $55 \mathrm{kDa}$ Ago2 protein levels accumulated upon proteasome inhibition, indicating that the turnover of both these proteins is proteasome dependent (Fig. 1E). However halfAgoz accumulation was greater than FL Ago2, signifying that halfAgo2 is a less stable protein that FL Ago2. We confirmed that accumulation of Ago2 protein was via a post-transcriptional mechanism as Ago2 transcript levels remain unchanged in $\mathrm{MG}_{132}$ treated samples (Fig. $\mathrm{S}_{1}$ ).

There is precedent in the literature that alternate forms of key proteins of the small RNA pathway have distinct roles to the full length form. Dicer, which is responsible for key steps of small RNA biogenesis, is proteolytically cleaved to form a highly-expressed, stable fragment (sDCR-1) that functions independently of full length Dicer, but has distinct regulatory functions in both the RNAi and miRNA pathways [19]. Our data suggests that halfAgoz is a stable truncated form of Agoz, and further studies are required to understand the function of halfAgoz and the implications this has on Agoz protein stability and RISC function.

Based on the size of the halfAgoz protein, we predict halfAgoz contains a functional PAZ domain, and therefore can potentially still bind small RNA molecules. This creates the possibility that halfAgo2 and FL Ago2 may be in competition for co-factor association and/or small RNA binding. Alternatively, halfAgoz may bind to a unique set of RNA molecules forming a distinct regulatory complex. This possibility would expand the range of traditional Agoz targets currently being investigated and may help to explain the number of predicted Agoz targets, as identified by high-throughput immunoprecipitation and RNA-protein crosslinking experiments, which have no associated miRNA binding sites [20].

As factors that influence Agoz and RISC stability are largely unknown, discovery of the novel mechanism behind halfAgoz production and what regulates this mechanism, is essential to fully understand the role of Ago2 stability in regulation of gene expression in both healthy tissues. Additionally, this will provide critical insights into how the Ago2 and RISC complex responds to stress, and how they become dysregulated in disease pathogenesis.

As there is increasing evidence that Agoz function, stability and turnover is regulated by post-translation modifications of the protein which are the result of a complex network of signalling pathways, it would be interesting to investigate if halfAgoz production is a consequence of such a modification. If so, it suggests halfAgoz formation is regulated in response to specific cellular or environmental triggers or stresses, as a mechanism to control RISC function in these particular situations.

halfAgo2 is a stable protein product which potentially has its own unique regulatory role. halfAgoz may play a role in RISC formation or activity, or alternatively, is capable of functioning independently from the miRNA pathway. Elucidating the production and function of halfAgo2 will further our understanding of Agoz stability and therefore miRNA expression and function. This can impact the development of future therapeutics which act to overexpress or inhibit miRNA function.

\section{Conclusions}

Our results indicate that unloaded human Agoz can form a stable protein product, of approximately $55 \mathrm{kDa}$, called halfAgo2. We propose that halfAgoz is produced from the post-transcriptional proteolytic cleavage of Agoz. We predict that halfAgoz is formed as a consequence of a mechanism which controls Agoz stability and turnover.

\section{Limitations}

Due to the nature of this study and the current tools available we are unable to isolate endogenous halfAgoz. This is because Ago family members are highly conserved. Therefore, the development of Agoz specific antibodies depends on a unique $\mathrm{N}$-terminal domain only present in Agoz. However, the N-terminal domain is also present in halfAgoz. It is therefore impossible to separate full length Ago2 from halfAgo2 via immunopre- 
cipitation, or other antibody based methods. It will therefore be necessary to accurately map what is encoded within the halfAgoz protein, to better understand its potential functions and so halfAgoz constructs can be generated. Alternatively, halfAgoz can be purified via size exclusion chromatography.

We propose that halfAgo2 is formed as a consequence of a proteolytic cleavage mechanism which controls Ago2 stability and turnover. There is mounting evidence that Ago2 stability is regulated by post-translational modifications (PTMs) of the protein. As many sites of PTMs have been predicted within the FL Agoz protein, only a few have known functional consequences [9]. Therefore it would be interesting to investigate if the formation of halfAgo2 was precipitated by such a modification. And if so, identify the signalling pathways involved in triggering this mechanism, and the specific stresses or environmental triggers required to prompt such a signalling cascade, as there is evidence that PTMs can control RISC activity and miRNA regulation efficiency in a signal dependent manner [11].

\section{Additional Information}

Methods and Supplementary Material

Please see https://sciencematters.io/articles/201811000001.

\section{Funding Statement}

G.H. is an ARC Future Fellow and this work was supported by the Australian Research Council Future Fellowship (FT110100455) and University of Technology Sydney startup fund.

Ethics Statement

Not Applicable.

\section{Citations}

[1] Kimberly Breving and Aurora Esquela-Kerscher. "The complexities of microRNA regulation: mirandering around the rules". In: The International fournal of Biochemistry and Cell Biology 42 (2010), pp. 1316-1329. DOI:

10.1016/j.biocel.2009.09.016. URL:

https://ac-els-cdn-com.ezproxy.lib.uts edu.au/S1357272509002726/1-s2.0-

S1357272509002726-

main.pdf?\%7B\%5C \%7Dtid=0f30107b-b912-

$421 \mathrm{~b}-\mathrm{a} 4 \mathrm{fb}-$

a 357eb3703db\%7B\%5C\&\%7Dacdnat $=1533879384 \%$ 7B\%5C_\%7Db524fa1e0eadddbec8784653e0bd9e9b.

[2] Kotaro Nakanishi. "Anatomy of RISC: how do small RNAs and chaperones activate Argonaute proteins?” In: Wiley Interdisciplinary Reviews (WIREs): RNA 7.October (2016), pp. 637-66o. Dor: $10.1002 /$ wrna. 1356

[3] Hotaka Kobayashi and Yukihide Tomari. "RISC assembly: Coordination between small RNAs and Argonaute proteins". In: Biochimica et Biophysica Acta (BBA) - Gene Regulatory Mechanisms 1859 (2016), pp. 71-81. DoI: $10.1016 / \mathrm{j}$.bbagrm. 2015.08.007. URL: https://ac-els-cdn-com.ezproxy.lib.uts . edu.au/S1874939915001844/1-s2.0S1874939915001844main.pdf?\%7B\%5C_\%7Dtid=a743760e-f732$11 \mathrm{e} 7-\mathrm{b} 993-$ 00000aab0f $27 \% 7 \mathrm{~B} \% 5 \mathrm{C} \& \% 7 \mathrm{Dacdnat}=1515718447 \%$ 7B\%5C \%7D91b1a5c82d19a131966b700086b64712.

[4] Marilena V. Iorio and Carlo M. Croce. "MicroRNA dysregulation in cancer: diagnostics, monitoring and therapeutics. A comprehensive review". In: EMBO Molecular Medicine 4.3 (Mar.
2012), pp. 143-159. DOI: $10.1002 /$ emmm . 201100209 . URL: http: //www. pubmedcentral.nih.gov/ articlerender.fcgi ? artid=3376845\%7B\%5C\&\% 7Dtool=pmcentrez\%7B\%5C\&\%7Drendertype $=$ abstract.

[5] Julia Winter and Sven Diederichs. "Argonaute proteins regulate microRNA stability: Increased microRNA abundance by Argonaute proteins is due to microRNA stabilization”. In: RNA Biology 8.6 (2011), pp. 1149-1157. DOI: 10.4161/rna.8.6.17665.

[6] Stefan Rüegger and Helge Großhans. "MicroRNA turnover: when, how, and why”. In: Trends in Biochemical Sciences 37.10 (Oct. 2012), pp. 436-446. DOI: 10.1016/j.tibs. 2012.07.002. unL: http : //www.ncbi.nlm.nih.gov/pubmed/22921610.

[7] Peter Smibert et al. "Homeostatic control of Argonaute stability by microRNA availability”. In: Nature Structural and Molecular Biology 20 (2013), pp. 789-795. DOI: $10.1038 / \mathrm{nsmb} .2606$. URL: http :

//www. nature.com/authors/editorial\%7B\%5C_ \%7Dpolicies/license.html\%7B\%5C\#\%7Dterms.

[8] Natalia J. Martinez and Richard I. Gregory. "Argonautez expression is post-transcriptionally coupled to microRNA abundance". In: RNA 19.5 (May 2013), pp. 605-612. DOI: 10.1261/rna.036434.112. uRL: http: //www.ncbi.nlm.nih.gov/pubmed/23485552.

[9] Joaquin Lopez-Orozco et al. "Functional analyses of phosphorylation events in human Argonaute 2". In: RNA 21.12 (2015), pp. 2030-2038. DoI: 10.1261/rna.053207.115. URL: http: //WwW.ncbi.nlm.nih.gov/pubmed/ 
26443379\%7B\%\%7D5Cnhttp:

//www.pubmedcentral.nih.gov/

articlerender. fcgi ?artid=PMC4647457.

[10] Hank H. Qi et al. "Prolyl 4-hydroxylation regulates Argonaute 2 stability". In: Nature 455.7211 (2008), pp. 421-424. DOI: 10. 1038 /nature07186. URL: www. nature.com/nature.

[11] Ryan J. Golden et al. "An Argonaute phosphorylation cycle promotes microRNA-mediated silencing”. In: Nature 542 (2017), pp. 197-202. Dor: 10 . 1038/nature21025. URL: http://www. nature.com/doifinder/10.1038/ nature 21025 .

[12] Umut Sahin et al. "Sumoylation of Human Argonaute 2 at Lysine-402 Regulates Its Stability”. In: PLOS ONE 9.7 (2014), e102957. DOI: 10.1371/journal pone. 0102957 . URL: wWw. plosone.org.

[13] Michael Johnston and Gyorgy Hutvagner. "Posttranslational modification of Argonautes and their role in small RNA-mediated gene regulation". In: Silence 2.1 (2011), p. 5. DOI: 10.1186/1758-907X-2-5. uRL: http : / /www . pubmedcentral.nih.gov/articlerender.fcgi? artid=3199228\%7B\%5C\&\%7Dtool=pmcent rez\%7B\% 5C\&\%7Drendertype $=$ abstract \%7B\%\%7D5Cnhttp : //wWw . silencejournal . com/content/2/1/5.

[14] Sebastian Petri et al. "Increased siRNA duplex stability correlates with reduced off-target and elevated on-target effects". In: RNA 17 (2011), pp. 737-749. DOI: 10 . 1261/rna . 2348111. URL: http://www. rnajournal.org/cgi/doi/10. 1261 /rna. 2348111 .

[15] Sabine Rüdel et al. "A multifunctional human Argonaute2-specific monoclonal antibody”. In: RNA 14 (2008), pp. 1244-1253. DOI: $10.1261 /$ rna . 973808. URL: https://www.ncbi.nlm.nih.gov/pmc/ articles/PMC2390805/pdf/1244.pdf.

[16] Pieter Bas Kwak and Yukihide Tomari. "The N domain of Argonaute drives duplex unwinding during RISC assembly”. In: Nature Structural and Molecular Biology 19.2 (Feb. 2012), pp. 145-151. DOI: 10 . 1038/nsmb. 2232. URL: http: //www.ncbi.nlm.nih.gov/pubmed/22233755.

[17] Kelley S. Yan et al. "Erratum: Structure and conserved RNA binding of the PAZ domain". In: Nature 427.6971 (2004), p. 265. DoI: $10.1038 /$ nature 02252 .

[18] Agnieszka Rybak et al. "The let-7 target gene mouse lin-41 is a stem cell specific E3 ubiquitin ligase for the miRNA pathway protein Ago2". In: Nature Cell Biology 11.12 (2009), pp. 1411-1420. DOI: $10.1038 /$ ncb1987.

[19] Ahilya N. Sawh and Thomas F. Duchaine. "A Truncated Form of Dicer Tilts the Balance of RNA Interference Pathways”. In: Cell Reports 4.3 (Aug. 2013), pp. 454-463. DOI: 10.1016/j . celrep. 2013.07.013. urL: http: //www.ncbi.nlm.nih.gov/pubmed/23933256.

[20] Sung Wook Chi et al. "Argonaute HITS-CLIP decodes microRNA-mRNA interaction maps”. In: Nature 460.7254 (2009), pp. $479-486$. 